\title{
Traduire
}

Revue française de la traduction

234 | 2016

La traduction, un sport de haut niveau

\section{Golf et traduction : de la page blanche à la balle blanche}

\section{Céline Graciet}

\section{(2) OpenEdition}

\section{Journals}

Édition électronique

URL : http://journals.openedition.org/traduire/792

DOI : $10.4000 /$ traduire.792

ISSN : 2272-9992

\section{Éditeur}

Société française des traducteurs

\section{Édition imprimée}

Date de publication : 15 juin 2016

Pagination : 24-27

ISSN : 0395-773X

\section{Référence électronique}

Céline Graciet, "Golf et traduction : de la page blanche à la balle blanche », Traduire [En ligne],

234 | 2016, mis en ligne le 15 juin 2018, consulté le 01 mai 2019. URL : http://journals.openedition.org/ traduire/792 ; DOI : 10.4000/traduire.792 


\section{Golf et traduction : de la page blanche à la balle blanche}

\section{Céline Graciet}

La pratique d'un sport est bien entendu utile quand on a la chance de se voir confier des documents à traduire sur ce thème. II y a quelques années, j'ai eu le bonheur de traduire des brochures destinées à la formation d'entraîneurs de foot, et cela reste mon projet préféré. Quoi de plus agréable que de mettre ses années d'expérience et de pratique d'un sport qu'on aime au service de son activité professionnelle? Je traduis aussi régulièrement les documents d'un nouveau club de golf, ce qui me ravit, car si le foot est mon sport de toujours, le golf est mon obsession actuelle, et j'aime à penser qu'il s'agit d'une forte formation professionnelle continue. En effet, la dimension psychologique de ce sport peut aider à élaborer des stratégies permettant à n'importe qui d'enrichir sa vie professionnelle, notamment dans les domaines suivants : concentration, discipline, gestion du stress, progression, compétences relationnelles et travail en équipe. Ce transfert de compétences ne se fait pas que dans un sens : mon métier de traductrice m'aide aussi dans certains aspects de mon sport.

\section{Quand la traduction se révèle utile sur le green}

J'en ai pratiqué beaucoup, des sports : foot, squash, pelote basque, course à pied et bien d'autres, mais le golf est le seul qui exige une concentration aussi intense sur une durée aussi longue. II est extrêmement difficile de rester concentrée pendant les quatre heures que dure en moyenne un match, et pourtant il le faut pour se préparer au prochain coup, analyser la situation et réfléchir à ses choix tactiques. J'ai remarqué dès le départ que j'avais moins de mal à me concentrer que la plupart de mes camarades et cela s'explique peut-être en partie par mon activité de traductrice. Voilà des années que je passe mes journées en apnée dans des textes ; je trouve donc le passage à un autre mode de concentration sur la durée relativement aisé. Mes deux principales occupations se rejoignent donc sur ce plan et se renforcent sans doute l'une l'autre ; d'ailleurs, je suis à peu près certaine que depuis que j'ai commencé le golf, j'ai beaucoup moins besoin de m'aérer l'esprit sur des sites web qui n'ont rien à voir avec la tâche 
en cours. Ou peut-être que le temps que je consacre à mon sport m'oblige à être plus productive quand je suis au travail...

Non pas que je manque de discipline : les échéances sont les échéances et le boulot passe en premier. Et cette discipline, cette maîtrise de soi, on la retrouve aussi dans le golf. La tentation de balancer ses clubs dans le plan d'eau le plus proche a beau être aussi fréquente que forte, c'est un geste que même l'Anglaise la plus flegmatique condamnerait sans hésiter d'un mouvement de tête imperceptible, mais réprobateur. On apprend vite à prendre sur soi et à contrôler des impulsions qui entraîneraient certes un soulagement immédiat, mais aussi une humiliation certaine. Après plusieurs après-midis passés à rater sa cible avec une admirable régularité, les petits problèmes techniques associés au quotidien de la traduction deviennent plus supportables. On apprend à hausser les épaules et à aller se faire un café en attendant que l'internet revienne ou que le client se décide à envoyer le document promis il y a plusieurs heures.

En effet, plus que tout autre, le golf est un sport où un mental solide est la clé de la réussite : impossible de pallier un manque de confiance ou une incapacité à anticiper un problème par un redoublement d'effort physique. II faut savoir se dominer pour rester calme et garder le cap, ce qui peut se révéler utile dans le travail quotidien. II m'est arrivé de douter de mes capacités face à un document particulièrement difficile requérant des recherches fouillées, des comparaisons terminologiques approfondies ou un travail stylistique particulier. Quand je me trouve dans une situation où je suis envahie par le doute, je reviens à l'attitude qui me permet de survivre à un match de golf dans la sérénité : au lieu de paniquer devant l'énormité de la tâche à accomplir (un parcours de 18 trous à apprivoiser), je me concentre sur le moment présent et le coup à jouer, sans m'inquiéter de ce qui va venir après ou de ce qui vient de se passer. Le document archicomplexe et mal écrit de 10000 mots est ainsi réduit à une phrase, puis une autre, puis une autre, ce qui rend le stress beaucoup plus gérable.

\section{De l'humilité dans son swing et au bureau}

Une bonne dose d'humilité est aussi essentielle si l'on souhaite faire des progrès au golf. C'est un sport extrêmement technique et très difficile, où tout se joue sur des millimètres et où les erreurs sont inévitables ; c'est en apprenant à les gérer et à les digérer que l'on s'améliore. Tout traducteur recevant une traduction revue et corrigée sait qu'il est parfois difficile d'accepter les critiques, surtout si elles sont justifiées. De même, le golf est tellement impitoyable qu'il est nécessaire d'adopter une attitude humble et d'accepter ses limites sous peine de finir en larmes dans un bunker. Bien sûr, nous cherchons tous à produire des traductions parfaites, mais la perfection absolue est un objectif dangereux et même contre-productif. Au golf comme au travail, il est beaucoup plus utile d'accepter ses erreurs et d'analyser honnêtement sa perfor- 
mance. Ainsi est-on plus susceptible d'éviter non seulement les boulettes, mais aussi les émotions négatives qui vont de pair, telles que la culpabilité, la perte de confiance en soi et la honte ; autant de barrières aux bonnes performances et aux progrès dans tous les domaines.

Autre source d'anxiété pour les traducteurs freelance : les clients. La plupart sont merveilleux, bien entendu, mais il peut arriver que certains d'entre eux soient plus difficiles ou que le courant passe mal. Et s'il est une chose que mes études ne m'ont pas du tout apprise, c'est la gestion de la relation client. Les rapports sont bien sûr différents avec les partenaires et adversaires sur un parcours de golf. Cependant, communiquer de manière efficace et harmonieuse avec une cliente issue d'une culture différente et qui ne comprend pas vraiment le processus de traduction n'est pas si éloigné des situations dans lesquelles je me trouve dans mon sport, notamment lors de compétitions où je passe mes journées en compagnie de parfaites inconnues. J'ai donc rapidement dû faire de gros progrès en diplomatie afin de trouver un terrain d'entente avec des joueuses aux opinions et aux expériences très éloignées des miennes ; heureusement, mon métier m'avait déjà préparée à échanger avec toutes sortes de personnes différentes et à me mettre à l'écoute des besoins d'autrui.

\section{La collaboration, encore et toujours}

Et bien sûr, ces qualités sont aussi utiles dans un petit club fauché comme le mien, dans lequel le travail en équipe est essentiel au quotidien. II suffit de s'investir un minimum pour réaliser à quel point le bon fonctionnement d'un club repose sur la bonne entente et la coordination de ses membres pour assurer la réalisation des multiples tâches indispensables. Ce genre de travail en équipe rappelle celui sur lequel repose la chaîne logistique de la traduction, dont les traductrices ne constituent qu'un maillon. Dans tous les sports, on apprend à trouver sa place au sein d'un collectif, à écouter, à contribuer, à négocier et parfois même à élever la voix pour se faire entendre. À force d'évoluer dans ces deux mondes, je ne cesse d'apprendre et de réapprendre l'art de la patience (c'est fou comme je l'oublie vite), de l'écoute et de la tolérance.

La traduction et le golf ont donc plus de choses en commun qu'on ne le croirait au premier abord, surtout sur le plan psychologique. L'autre avantage du golf, c'est bien sûr son impact sur la santé mentale et physique. Passer huit heures par jour voûtée sur son clavier, ce n'est idéal ni pour le dos, ni pour l'humeur. Un neuf trous rapide après ou avant le travail, c'est idéal pour s'aérer, se dégourdir les jambes et recharger ses batteries. Dans mon quotidien de traductrice, j'apprécie doublement le fait de prendre un grand bol d'air frais dans un environnement vert et ces moments passés loin d'un écran m'aident à supporter les longues heures à tapoter sur mon clavier. Je suis plus productive, quoi. Le golf n'est bien sûr pas le seul sport à avoir cet effet bénéfique ; n'importe quelle activité qui change les idées et oblige à bouger 
est à recommander à tous les travailleurs indépendants, qui ont facilement tendance à se laisser happer par leur carrière au détriment de leur bien-être mental et physique. Quelle est la vôtre?

Céline Graciet est traductrice de l'anglais vers le français depuis 16 ans. Elle travaille pour des clients dans des secteurs variés, dont le développement international, la protection de l'environnement, le marketing, le sport, le fitness et le bodybuilding. Se sentant coupable de passer des journées heureuses à exercer une profession aussi enrichissante que passionnante, elle a décidé il y a quatre ans de commencer à jouer au golf pour introduire une source de frustration intense dans son existence et parvenir ainsi à un meilleur équilibre dans sa vie par trop paisible.

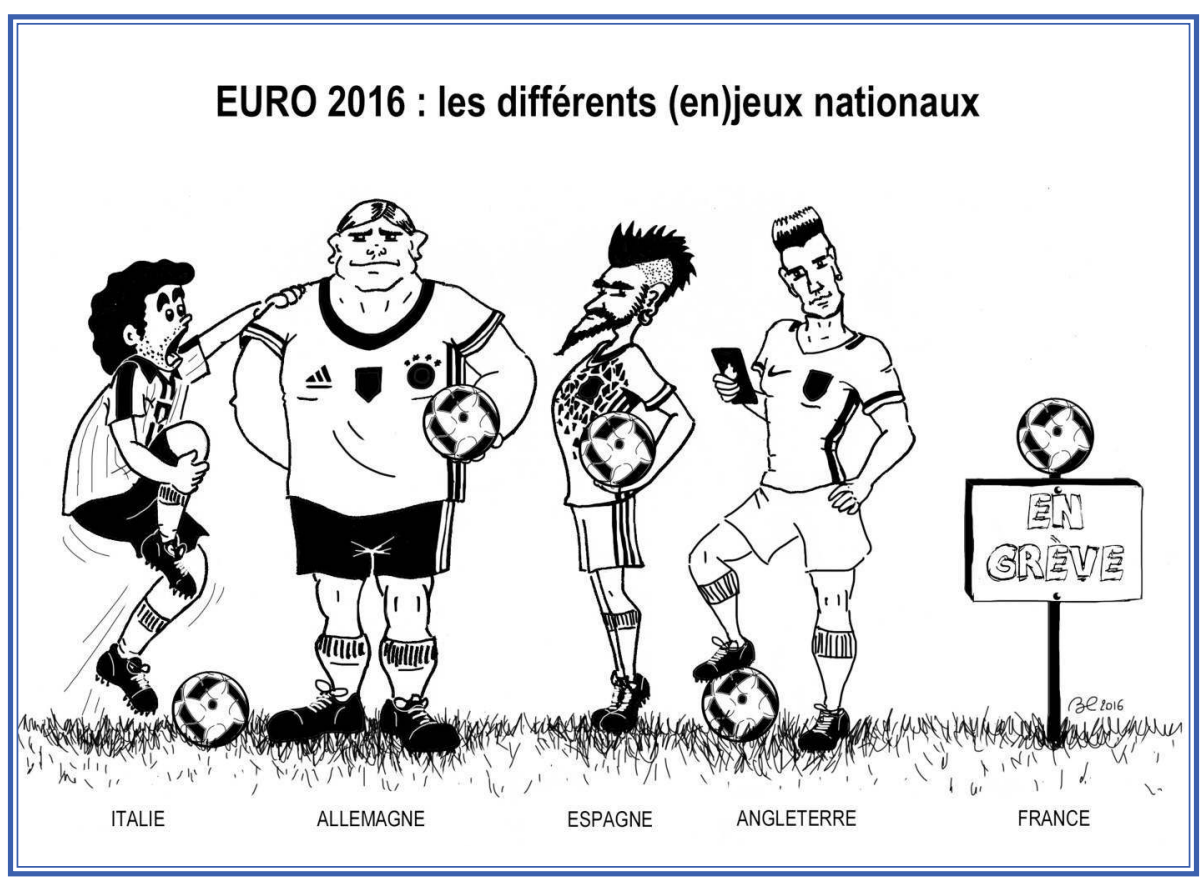

